\title{
SUR LES ÉQUATIONS DE LA DYNAMIQUE
}

\author{
PAR
}

\section{R. LIOUVILLE \\ à PARIs.}

\section{Introduction.}

L'Académie des Sciences de Paris avait proposé, comme sujet d'un prix à décerner en 1894 , l'étude des intégrales algébriques des équations de la dynamique et particulièrement des intégrales quadratiques. Le travail, que j’avais présenté à ce concours et auquel une mention honorable a été accordée, se composait de deux parties. La première, se rapportant à une certaine interprétation des équations de la dynamique et surtout à l'étude de leurs intégrales quadratiques, est reproduite dans le présent Mémoire, sans autre changement que la suppression de quelques détails, mieux à leur place parmi d'autres recherches.

En ce qui concerne les intégrales quadratiques, je n'ai point fait porter mes efforts sur toutes les catégories dans lesquelles ces intégrales peuvent être rangées.

L'objet principal des premiers paragraphes de ce Mémoire est, au contraire, la définition précise d'une certaine espèce d'intégrales quadratiques, à laquelle celle des forces vives appartient toujours lorsqu'elle existe et qui jouit de propriétés très distinctes.

Le sujet ainsi limité se trouve en liaison étroite avec un autre problème, déja étudié sur certains points par plusieurs auteurs, c'est celui de la conservation des trajectoires. On sait en effet que, dans certains cas, les équations de la dynamique admettent des transformations, différentes de celles qui consistent dans un simple changement des variables 
et susceptibles d'ètre employées sans altération des trajectoires. Le mouvement sur ces trajectoires est en général modifié, mais il peut aussi ne pas l'être et l'on construit sans peine des exemples pour lesquels se produit ce fait assez singulier. Quoiqu'il en soit à cet égard, les problèmes admettant les transformations indiquées font aussi connaître, parmi les intégrales du second degré des équations de la dynamique, des classes douées de caractères spéciaux. Il faut toutefois séparer, dans toute cette question, deux points de vue presque opposés, car on peut faire l'une ou l'autre des deux hypothèses suivantes:

A. I re hypothèse. Les forces sont nulles, ou bien elles dérivent d'un potentiel et l'énergie est une constante donnée; il est bien connu qu'à ces deux conditions répondent des équations de même espèce. L'étude des transformations conservant les trajectoires n'est alors rien autre chose qu'une question relutive aux équations différentielles linéaires, à une seule variable; c'est ce que peuvent établir les $\S \mathrm{I}, 2$ et 3 de ce travail et voici la question dont il s'agit:

On donne un système d'équations différentielles linéaires,

$$
\begin{array}{r}
d z-\sum_{(h)} z^{(h)} d x_{h}=0, \\
d z^{(i)}+\sum_{(h, k)} p_{i . k}^{(h)} z^{(h)} d x_{k}=0,
\end{array}
$$

dans lequel les inconnues sont $z, z^{(1)}, \ldots, z^{(n)}$; les coefficients $p_{i . k}^{(h)}$ sont des fonctions données de $m$ variables $x_{1}, x_{2}, \ldots, x_{m}$, liées elles-mêmes à l'une d'entre elles, par $m-$ I équations qui ne sont pas connues. Comment doivent ètre choisies les fonctions données $p_{i . k}^{(h)}$, afin que le système linéaire précédent admette une, deux ou plusieurs intégrales, contenant au second degré les inconnues $z^{(h)}$ et indépendantes des liaisons supposées entre les $x_{i}$ ?

Je démontre que, s'il existe une intégrale de cette espèce, il y a un problème de dynamique correspondant aux équations linéaires mentionnées; s’il existe deux intégrales, il y en a $m$ en général, distinctes ou réductibles; chacune d'elles définit un mouvement et, lorsqu'on passe de l'un à l'autre de ceux-ci, les trajectoires ne sont pas changées. De plus, les équations de ces mouvements admettent des intégrales quadratiques, d'ordinaire au nombre de $m,(\$ 3)$. 
On peut imaginer que le système ( $\mathrm{I}$ ) n'admette aucune intégrale quadratique; cela n'empêche point qu'il lui corresponde des équations différentielles, analogues à celles de la dynamique et, comme elles, s'offrant sans cesse les mèmes, quel que soit le choix des variables $x_{1}, x_{2}, \ldots, x_{m}$. Je dirai que ces équations ont même aspect que celles de la dynamique; certaines conclusions relatives à ces dernières conviennent aussi aux équations de même aspect, dont la généralité cependant est plus grande. On voit qu'au contraire, les intégrales quadratiques dont il vient d'être question, sont, d'après leur nature mème, réservées aux équations de la dynamique proprement dites.

Il serait naturel d'étendre ces recherches en proposant d'attribuer au système (I) une ou plusieurs intégrales linéaires, une ou plusicurs intégrales de degré supérieur à deux; ${ }^{1}$ je laisse les intégrales linéaires, pour lesquelles le résultat est simple et sera développé dans une autre occasion; quant à celles de degré supérieur à deux, on serait tenté de penser qu'elles n'existent pas, si l'on n'avait pu former des exemples pour lesquels se présentent $m$ intégrales quadratiques; or celles-ci permettent de construire des intégrales, de degré supérieur à deux et même assez variées. La question est alors de. savoir s’il y a, pour le système ( 1 ), des intégrales entières, hormis celles qui résultent ainsi des combinaisons de quelques autres, soit linéaires, soit quadratiques. Cela est impossible et la démonstration se fait en quelques mots $(\$ 4)$, sous la réserve d'un cas exceptionnel, dont l'examen complet ne m'a pas paru nécessaire en ce moment.

La recherche qui reste à faire est celle des problèmes de dynamique pour lesquels les équations linéaires correspondantes (I) admettent, soit une intégrale linéaire, soit une seconde intégrale quadratique. Sur ce point, voici les résultats contenus dans ce mémoire.

Comme conséquence des relations précédemment établies, j'indique d'abord une solution assez étendue et qui convient quel que soit le nombre des variables $x_{h}$. Afin qu'elle se présente, il faut et il suffit que les deux formes quadratiques conjuguées, je veux dire correspondant aux mèmes trajectoires, puissent être simultanément réduites à ne contenir

1 Les équations de la dynamique correspondantes auraient aussi des intégrales de ces mêmes degrés et d'une nature spéciale. 
R. Liouville.

que les carrés des différentielles; il entre, dans les coefficients de ces deux formes, $m$ fonctions arbitraires et chacune de celles-ci dépend d'une variable unique. La solution ainsi trouvée offre une apparente analogie avec l'une de celles qui, liées à l'étude générale des intégrales quadratiques, sont depuis longtemps connues, mais elle en est au fond tout à fait différente et constitue un cas d'intégration nouveau, $(\$ 5)$.

$\mathrm{Au}$ reste, à toute solution connue du problème, le nombre des variables étant $m$, correspondent d'autres solutions, pour lesquelles ce nombre est un multiple de $m$ et qui se déduisent de la première sans aucun calcul; ce théorème est l'objet $d u \S 6$.

B. $2^{d}$ hypothèse. Dans les problèmes de mécanique considérés, les forces appliquées ne sont pas nulles; ce sont des fonctions quelconques des coordonnées $x_{1}, x_{2} \ldots, x_{n}$ et, lorsqu'elles dérivent d'un potentiel, la constante de l'énergie doit rester arbitraire. Si l'on veut étudier deux problèmes de cette espèce, différents et pour lesquels les trajectoires soient cependant les mêmes, on rencontre d'abord une proposition, dûe à M. Painlevé: il existe en général, pour chacun des problèmes une intégrale quadratique, $(\$ 7)$, une exception toutefois ayant lieu quand, après avoir fait évanouir les forces, les deux systèmes auxquels on parvient sont de la catégorie examinée plus haut, $(A)$.

La démonstration donnée $(\$ 7)$ se déduit des seuls éléments dont nous avons déjá fait usage; mais le théorème acquiert par ce moyen une nouvelle signification et un degré de généralité tout différents; c'est ce que je voudrais expliquer ici, car cela est essentiel pour présenter la question sous son jour véritable. J'ai déjà fait observer, au sujet de la première hypothèse, que certains systèmes d'équations différentielles ne sont pas susceptibles de définir l'ensemble des trajectoires dans un problème de mécanique proprement dit, mais offrent cependant le même aspect que les équations de ces trajectoires et jouissent d'une généralité beaucoup plus grande. La même chose a lieu dans le cas actuel et pour les mêmes raisons. L'intégrale quadratique, dont nous avons démontré l'existence, convient à ces problèmes plus généraux que ceux de la mécanique, dans les circonstances mèmes qui étaient admises pour ces derniers; cette existence n'est donc nullement liée, comme elle l'était dans la première hypothèse, à celle d'un véritable problème de mécanique correspondant aux équations étudiées; les deux faits sont au contraire 
distincts, bien que sans doute leur réunion puisse motiver des conclusions spéciales.

Il semble certain qu'entre les systèmes d'équations différentielles appartenant à des problèmes de dynamique et les systèmes plus généraux, de même aspect, il $y$ a des rapprochements très nombreux. Le $\S 8$ de ce mémoire est consacré à en indiquer un, presque évident: s'il arrive qu'un problème de dynamique admette une intégrale, rationnelle à l'égard des vitesses, il y a un système, de mème aspect, possédant une intégrale entière par rapport à ces mêmes quantités.

J'ajoute que les éléments employés dans toute cette théorie sont susceptibles d'applications assez différentes; c'est ainsi que d'une remarque faite au $\$ 2$ résulte, pour tout changement des variables $x_{1}, x_{2}, \ldots, x_{m}$, le moyen de construire les invariants non pas seulement des équations de la dynamique, mais aussi ceux, bien moins accessibles, des équations de mème aspect, soustraites à toute restriction.

La seconde partie du travail que j'avais présenté à l'Académie des Sciences de Paris concernait le problème de la rotation d'un corps solide autour d'un point fixe; ce sera le sujet d'un prochain Mémoire.

\section{CHAPITRE I.}

§ 1. Equations du mouvement, quand il y a une intégrale des forces vives, dont la coinstante est donnée. Systèmes linéaires associés.

Equations du second ordre plus générales que celles de la dynamique.

Quand un problème de dynamique admet l'intégrale des forces vives, il convient souvent de regarder comme une donnée de la question la valeur de la constante qui représente l'énergie. Le problème posé de cette manière équivaut, on le sait, à celui des géodésiques généralisées, et, comme il est d'une importance et d'une simplicité particulière, c'est à lui que se rapporteront un grand nombre des résultats suivants, notamment 
ceux qui font l'objet de ce paragraphe. Si l'on représente par $T$ la demi-somme des forces vives, par $x_{1}, x_{2}, \ldots, x_{m}$, les paramètres, en nombre quelconque, servant à définir la position du système matériel à l'instant considéré, par $t$ le temps compté depuis une origine arbitraire, dans la formule

$$
2 T d t^{2}=\sum_{(i . k)} e_{i, k} d x_{i} d x_{k},
$$

qui détermine $T$, les coefficients $e_{i, k}$ sont des fonctions quelconques de $x_{1}, x_{2}, \ldots, x_{m}$. Les équations $\mathrm{du}$ mouvement,

$$
\frac{d x_{i}}{d t}=x_{i}^{\prime}, \quad \frac{d}{d t}\left(\frac{\partial T}{\partial x_{i}^{\prime}}\right)-\frac{\partial T}{\partial x_{i}}=0,
$$

se développent alors ainsi

$$
\sum_{(k)} e_{i, k} \frac{d^{2} x_{k}}{d t^{2}}+\sum_{\left(h, h^{\prime}\right)}\left(\frac{\partial e_{i, h^{\prime}}}{\partial x_{h}}-\frac{1}{2} \frac{d e_{h . h^{\prime}}}{\partial x_{i}}\right) \frac{d x_{h}}{d t} \frac{d x_{h^{\prime}}}{d t}=0
$$

et leur étude se rattache, comme on le va voir, ̀̀ celle d'un certain système d'équations différentielles linéaires.

Considérons en effet le système:

$$
d z-\sum_{(h)} z^{(h)} d x_{h}=0
$$

$$
d z^{(\mathfrak{h})}+\sum_{(h, \boldsymbol{k})} p_{i, k}^{(h)} z^{(h)} d x_{k}=0
$$

dont les coefficients sont des fonctions arbitraires des variables $x_{1}, x_{2}$, $\ldots, x_{m}$; je suppose ces dernières liées par $m-\mathrm{I}$ relations, qui d'abord ne sont pas données et je regarde $z^{(1)}, z^{(2)}, \ldots, z^{(m)}$ et $z$ comme des inconnues satisfaisant aux équations linéaires (2). La dernière, $z$, qui joue un rôle prépondérant, nullement influencé d'ailleurs par le choix des variables $x_{1}, \ldots, x_{m}$, sera désignée sous le nom d'inconnue principale; les autres sont les inconnues auxiliaires. J'imagine que l'on veuille, dans les équations (2), isoler l'inconnue principale. Les relations établies entre les $x_{i}$ étant arbitraires, des différentiations, en général au nombre de $m$, permettent d'éliminer les inconnues auxiliaires et laissent une équation différentielle, d'ordre $m$, faisant connaitre $z$. Mais les relations adoptées peuvent être telles que l'ordre de cette équation soit abaissé. Pour qu'il 
devienne égal à deux, les variables $x_{i}$ doivent être assujetties à des conditions, qui les définissent d'une façon complète. En effet de la première égalité (2), on déduit

$$
d^{2} z-\sum_{(i)} d z^{(i)} d x_{i}-\sum_{(h)} z^{(h)} d^{2} x_{h}=0,
$$

ou bien, d'après les égalités du même groupe,

$$
d^{2} z-\sum_{(h)} z^{(h)}\left[d^{2} x_{h}-\sum_{(i, k)} p_{i, k}^{(h)} d x_{i} d x_{k}\right]=0^{\bullet}
$$

et le résultat cherché sera obtenu pourvu que ces équations différentielles,

$$
\frac{d^{2} x_{h}-\sum_{(i, k)} p_{i, k}^{(i)} d x_{i} d x_{k}}{d x_{h}}=\frac{d^{2} x_{h^{\prime}}-\sum_{(i, k)} p_{i, h}^{\left(h^{\prime}\right)} d x_{i} d x_{k}}{d x_{k^{\prime}}}
$$

soient vérifiées, quels que soient les indices $h$ et $h^{\prime}$. Cela étant, on en conclut la relation

$$
d x_{h} d^{2} z-d z\left[d^{2} x_{h}-\sum_{(i, k)} p_{i, k}^{(h)} d x_{i} d x_{k}\right]=0
$$

où l'indice $h$ est à volonté. Le lien étroit qui existe entre ces équations et les précédentes ( $\mathrm{I}$ ) s'aperçoit sans peine. Ayant pris pour $t$ l'inconnue $z$, il suffit que les fonctions $p_{i . k}^{(h)}$ soient déterminées comme il convient pour que les systèmes d'équations (5) et (I) se confondent entièrement. Je dirai alors que le système linéaire (2) leur est associé. On voit que les $p_{i . k}^{(h)}$ ne sont pas des fonctions quelconques des variables $x_{1}, x_{2}, \ldots, ., x_{m}$, quand même les coefficients $e_{i . k}$ seraient regardés comme arbitraires. Mais, que l'on suppose au contraire, entre $p_{i, k}^{(h)}$ et les $x_{h}$, des relations à volonté: les équations (5) et, par suite, (4) conservent le même aspect; la seule différence consiste en ce qu'il cesse d'y avoir des coefficients $e_{i . k}$ correspondants. Dans ce qui va suivre, j'aurai souvent à considérer les équations (4) dans toute leur généralité, mais j'imposerai d'ordinaire aux coefficients $p_{i, k}^{(h)}$, que ces équations (4) ne déterminent pas d'une façon complète, une condition importante, c'est qu'il existe une fonction $\hat{o}$, dont les dérivées partielles s'expriment ainsi

$$
\frac{\partial \log \delta}{\partial x_{k}}=-\sum_{(i)} p_{i . k}^{(i)}
$$


R. Liouville.

Gràce à cette hypothèse, si le système (4) et la fonction $o$ sont donnés, toutes les quantités $p_{i . k}^{(h)}$ sont également connues. Dans ce cas encore, je dirai que les équations (4) et le système linéaire (2) sont associés.

On peut encore se représenter d'une autre manière le lien qui rattache les équations (4) ou (5) au système linéaire associé. Considérons, sous la forme

$$
\sum_{(h)} q_{h} z^{(h)}=\text { constante, }
$$

une intégrale de ce système, pour un choix quelconque des liaisons établies entre les variables $x_{i}$; il est clair que les fonctions $g_{i}$ peuvent étre regardées comme des inconnues nouvelles, satisfaisant aux équations linéaires de ce système

$$
d q_{i}-\sum_{(h . k)} p_{h . k}^{(i)} q_{h} d x_{k}=\mathrm{o}
$$

adjoint à (2). Pour définir les $x_{i}$, je suppose, outre les équations (2) et $\left(2^{\prime}\right)$ les suivantes

$$
\frac{d x_{i}}{d z}=q_{i}
$$

on vérifie sans peine qu'elles entrainent les équations (5) et n'en exigent aucune autre. En raison de sa simplicité, j'omets cette vérification.

La nouvelle manière, ainsi obtenue, de rattacher les équations (4) au système linéaire (2) ou plutòt à son adjoint, est, à quelques égards, plus naturelle que la première, mais elle met moins en évidence l'un des principaux caractères de cette connexion, son invariance pour tous les choix possibles des variables $x_{1}, x_{2}, \ldots, x_{m}$. Au reste, alors que le système (2') est en relations plus directes avec les équations de la dynamique, telles que IAAgrange les a construites, le système (2) ist au contraire plus étroitement lié aux équations d'Hamiton, c'est la conclusion bien aisée à déduire des remarques précédentes.

Lorsqu'il existe des coefficients $e_{i^{\prime}, k^{\prime}}$, leurs relations avec les quantités $p_{i . k}^{(h)}$ peuvent être mises sous cette forme

$$
\frac{\partial e_{i, k}}{\partial x_{i^{\prime}}}+\sum_{(h)}\left(e_{i . h} p_{k, i^{\prime}}^{(h)}+e_{h . k} p_{i . i^{\prime}}^{(h)}\right)=0
$$


En effet, après avoir remplacé $z$ par $t$ dans les équations (5), on voit que pour les faire coincider avec (I), il est nécessaire et suffisant que ces identités

$$
\sum_{\left(h, h^{\prime}\right)} d x_{h} d x_{h}\left[\frac{\partial e_{i, h^{\prime}}}{\partial x_{h}}-\frac{\mathrm{I}}{2} \frac{\partial e_{h, h^{\prime}}}{\partial x_{i}}+\sum_{(k)} p_{h, h^{\prime}}^{(k)} e_{i, k}\right]=0
$$

soient vérifiées. Comme les indices $h$ et $h^{\prime}$ reçoivent toutes les valeurs $\leqq m$, chaque produit $d x_{h} d x_{h^{\prime}}$ a pour facteur

$$
\left(\frac{\partial e_{i, h^{\prime}}}{\partial x_{h}}-\frac{\mathrm{I}}{2} \frac{\partial e_{h, h^{\prime}}}{\partial x_{i}}+\sum_{(k)} p_{h . h^{\prime}}^{(k)} e_{i . k}\right)+\left(\frac{\partial e_{i, h}}{\partial x_{h^{\prime}}}-\frac{\mathrm{I}}{2} \frac{\partial e_{h^{\prime}, h}}{\partial x_{i}}+\sum_{(k)} p_{h^{\prime}, h}^{(k)} e_{i . k}\right) .
$$

Mais la seconde parenthèse a été déduite de la première en permutant $h$ et $h^{\prime}$ et la somme des deux devant être nulle, on en conclut

$$
\frac{\partial e_{i, h^{\prime}}}{\partial x_{h}}+\frac{\partial e_{i . h}}{\partial x_{h^{\prime}}}-\frac{\partial e_{h . h^{\prime}}}{\partial x_{i}}+2 \sum_{(k)} p_{h^{\prime}, h}^{(k)} e_{i . k}=0
$$

Dans cette identité, je permute $i$ avec $h$, puis avec $h$, ce qui donne les deux suivantes

$$
\begin{aligned}
& \frac{\partial e_{h . h^{\prime}}}{\partial x_{i}}+\frac{\partial e_{i . h}}{\partial x_{h^{\prime}}}-\frac{\partial e_{i, h^{\prime}}}{\partial x_{h}}+2 \sum_{(k)} p_{i . h^{\prime}}^{(k)} e_{k . h}=0, \\
& \frac{\partial e_{i, h^{\prime}}}{\partial x_{h}}+\frac{\partial e_{h . h^{\prime}}}{\partial x_{i}}-\frac{\partial e_{h . i}}{\partial x_{h^{\prime}}}+2 \sum_{(k)} p_{i . h}^{(k)} e_{k, h^{\prime}}=0 .
\end{aligned}
$$

L'ensemble (9, 10) équivaut aux relations (7) et le laisse d'abord reconnaître. On voit de plus que les $p_{i . k}^{(h)}$ sont toujours déterminés quand les $e_{i . k}$ le sont eux-mêmes, pourvu que le déterminant symétrique de ces derniers diffère de zéro; or ceci est une condition remplie par les équations de la dynamique.

Soit $\Delta$ ce déterminant: il résulte immediatement des équations (7), eu égard aux propriétés bien connues des déterminants

$$
d\lrcorner+2 \Delta \sum_{(i, h)} p_{i . k}^{(l)} d x_{i}=0
$$

et, d'après les formules (6),

$$
\Delta=c \hat{\sigma}^{2},
$$

$c$ désignant une constante arbitraire. 
Soient $E_{i, k}$ des fonctions ainsi définies

$$
\sum_{(i)} E_{i . k} c_{i, k}=\mathrm{I}, \quad \sum_{(i)} E_{i . h} e_{i, k}=\mathrm{O}
$$

Voici, en tenant compte de (7), la conséquence évidente des égalités ( I 3),

$$
d E_{i . k}-\sum_{\left(h, i^{i}\right)}\left(E_{i, h} p_{i^{\prime}, h}^{(k)}+E_{h . k} p_{h, i^{\prime}}^{(i)}\right) d x_{i^{\prime}}=0
$$

permettant de déterminer les $E_{i . k}$ sans aucun intermédiaire.

Réciproquement, si des inconnues doivent satisfaire aux équations (14), il est toujours permis de les supposer liées aux coefficients $e_{i . k}$ par les relations (I 3); la façon symétrique dont les systèmes (14) et (7) se correspondent suffit pour le faire voir.

\section{§ 2. Equations différentielles d'ordre supérieu' formant un système incomplet. Invariants des équations de la dynamique ou des équations générales de même aspect.}

Nous avons dit $\S$ I qu'aux équations de cette espèce

$$
\frac{d^{2} x_{h}-\sum_{(i, k)} p_{i, k}^{(h)} d x_{i} d x_{k}}{d x_{h}}=\frac{d^{2} x_{h}-\sum_{(i, k)} f^{\left(h_{i, k}^{\prime}\right)} d x_{i}, l x_{i}}{d x_{h}}
$$

qui comprennent comme cas particulier les équations de la dynamique, est associé un système linéaire, toujours le mème quel que soit le choix des variables $x_{i}$. On peut à ce dernier rattacher toute une série de groupes d'équations entre les $x_{i}$; ces équations sont analogues à (1), mais d'ordre supérieur à deux; elles forment des systèmes incomplets, où le nombre des relations distinctes est inféricur à $m-\mathrm{I}$.

Il n'y a, pour les obtenir, qu'à différentier un nombre quelconque de fois la première équation $\left(2 \oint 1\right.$ ) et a supposer que les variables $x_{i}$ sont assujetties à des conditions telles que toutes les équations ainsi construites ne soient pas indépendantes. 
Par exemple, si l'on pose pour abréger

$$
\begin{gathered}
D d x_{h}=d^{2} x_{h}-\sum_{(i, k)} p_{i . k}^{(h)} d x_{i} d x_{k} . \\
D^{2} d x_{h}=D . D d x_{h}=d D d x_{h}-\sum_{(i . k)} p_{i, k}^{(h)}\left(d x_{k} D d x_{i}+d x_{i} D d x_{k}\right), \\
\text { etc., }
\end{gathered}
$$

de la première équation $(2 \oint \mathrm{I})$ il résulte

$$
\begin{aligned}
& d^{2} z-\sum_{(h)} z^{(h)} D d x_{h}=0, \\
& d^{3} z-\sum_{(h)} z^{(h)} D^{2} d x_{h}=0 ;
\end{aligned}
$$

ces relations et celle d'où l'on est parti,

$$
d z-\sum_{(h)} z^{(h)} d x_{h}=0
$$

cesseront d'étre indépendantes, si l'on imagine que les variables $x_{i}$ satisfassent à ces identités

$$
\left|\begin{array}{ccc}
d x_{h} & d x_{h^{\prime}} & d x_{h^{\prime \prime}} \\
D d x_{h} & D d x_{h^{\prime}} & D d x_{h^{\prime \prime}} \\
D^{2} d x_{h} & D^{2} d x_{h^{\prime}} & D^{2} d x_{h^{\prime \prime}}
\end{array}\right|=\text { o, }
$$

dont $m-2$ sont seules distinctes. Lorsqu'elles ont lieu, l'inconnue principale $z$ satisfait à une équation différentielle du $3^{\mathrm{e}}$ ordre, qu'on peut représenter ainsi

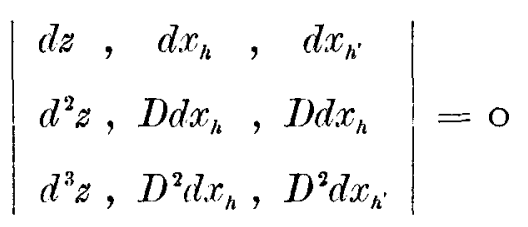

Rien n'empêche de parvenir aux équations (3) et (4), en les rattachant au système $(2 ', \S 1)$. Elles expriment en effet que, $z$ étant donnée, il $\mathrm{y}$ a entre les $z^{(i)}$ deux relations entièrement connues et non davantage. Or l'équation suivante

$$
\sum_{(h)} q_{h} z^{(h)}=c
$$


peut être substituée à l'une de celles qui constituent le système (2), puisqu'elle en est une intégrale et, les mêmes conditions étant imposées, les équations (4) sont remplacées par celles-ci

$$
\left|\begin{array}{ccc}
c & q_{h} & q_{h^{\prime}} \\
d z & d x_{h} & d x_{h^{\prime}} \\
d^{2} z & D d x_{h} & D d x_{h^{\prime}}
\end{array}\right|=0
$$

dans lesquelles la constante $c$ est arbitraire. J'ajoute que les équations du second ordre étudiées au $\$$ I font connaitre une solution particulière des équations (4) ou (6) de ce paragraphe.

J'indiquerai une seule application des systemes in omplets tels que (3); elle est relative au dernier d'entre eux. De l'égalité

$$
d z-\sum_{(h)} z^{(h)} d x_{h}=0
$$

peuvent ètre déduites, on le sait, les suivantes

$$
d^{2} z-\sum_{(h)} z^{(h)} D d x_{h}=0, \ldots, \quad d^{m} z-\sum_{(h)} z^{(h)} D^{m} d l x_{h}=0
$$

mais, afin qu'elles cessent d'être distinctes, il est nécessaire et suffisant qu'il y ait entre les $x_{i}$ une relation unique, d'ordre $m$,

$$
\left|\begin{array}{c}
d x_{h} \\
D d x_{h} \\
\cdot \\
D^{m} d x_{h}
\end{array}\right|=0
$$

celle-ci constitue seule l'un des groupes d'équations liés d'une manière invariante aux systèmes linéaires $(2)$ et $\left(2^{\prime}\right) \S 1$ et, par suite, aux équations (4) et (5) du même paragraphe. Si donc on considère, dans cette relation, l'ensemble des termes ne dépendant que des différentielles du premier ordre, cet ensemble est un covariant des équations (4) § I. II fait connaitre un moyen effectif de construire les invariants de ces équations si générales, dans lesquelles toutes celles de la dynamique se trouvent comprises. 
Je me borne à ces observations sur un sujet dont l'étude détaillée n'est pas indispensable pour la suite du présent Mémoire.

\section{§ 3. Conditions spéciales aux équations de la dynamique. Pro- priétés correspondantes les systèmes linéaires associés. Géuéralisation du problème de M. Dini.}

Je conserve les notations $\mathrm{du} \S_{\mathrm{I}}$ et, admettant que les équations différentielles

$$
\frac{d^{2} x_{h}-\sum_{(i, k)} p_{i, k}^{(h)} d x_{i} d x_{k}}{d x_{k}}=\frac{d^{2} x_{h^{\prime}}-\sum_{(i, k)} p_{i, k}^{\left(h^{\prime}\right)} d x_{i} d x_{k}}{d x_{h^{\prime}}}
$$

sont celles d'un problème de dynamique proprement dit, je considère les identités $(7) \S \mathrm{I}$,

$$
\frac{\partial e_{i . k}}{\partial x_{i^{\prime}}}+\sum_{(h)}\left(p_{k, i^{\prime}}^{(h)} e_{i . h}+p_{i . i^{\prime}}^{(h)} e_{k, h}\right)=\mathrm{o}
$$

qui traduisent cette hypothèse. Elles ont, pour le système linéaire

$$
d z-\sum_{(h)} z^{(h)} d x_{h}=0, \quad d z^{(i)}+\sum_{(h, k)} p_{i . h}^{(h)} z^{(h)} d x_{k}=0
$$

associé à (I), une signification très visible: elles expriment qu'il possède, quelles que soient les relations établies entre les $x_{i}, m$ solutions telles que les expressions correspondantes de $d z$ verifient la condition suivante

$$
\sum_{\langle h\rangle} d z_{h}^{2}=\sum_{(i . k)} e_{i . k} d x_{i} d x_{k}
$$

Je déduis en effet des relations (3) celles qui sont satisfaites par les produits $z^{(i)} z^{(k)}$ et je trouve

$$
d\left(z^{(i)} z^{(k)}\right)+\sum_{\left(h, i^{\prime}\right)}\left(p_{k, i^{\prime}}^{h)} z^{(i)} z^{(h)}+p_{i, i^{\prime}}^{(h)} z^{(k)} z^{(h)}\right) d x_{i^{\prime}}=0 ;
$$

les équations (2) en définissent précisément une solution, indépendante des liaisons établies entre les $x_{i}$,

$$
e_{h . k}=c \cdot z^{(h)} z^{(k)}
$$

et de laquelle on conclut la formule (4). 
Le plus souvent d'ailleurs cette solution est unique, abstraction faite de l'indéterminée $c$ qui la multiplie. Hais les conditions (2) sont susceptibles d'autres interprétations. Elles expriment que le système (3) possède une intégrale du second degré,

$$
\sum_{\left(h . h^{\prime}\right)} E_{h . h^{\prime}} \tilde{z}^{(h)} z^{\left(h^{\prime}\right)}=\text { constante, }
$$

ou bien qu'il y a, pour le système adjoint $a(3)$, une intégrale quadratique

$$
\sum_{\left(h . h^{\prime}\right)} e_{h . h^{\prime}} q_{h} q_{h^{\prime}}=\text { constante, }
$$

quelles que soient les relations établies entre les $x_{i}$. La constatation est des plus simples et ne mérite pas qu'on s'y arrète, mais le fait est digne d'intérêt, à cause de ses conséquences.

Les conditions (2) étant supposées remplies, les relations (I 4) $\$$ I,

$$
\frac{\partial E_{i, \boldsymbol{k}}}{\partial x_{i^{\prime}}}-\sum_{(h)}\left(p_{h, h^{(k)}}^{(k)} E_{i . h}+p_{h, i}^{(i)} E_{h . . h}\right)=\mathrm{o},
$$

sont aussi satisfaites; les notations suivantes

$$
E_{i . k}=f_{i . k} \cdot 0^{\frac{-2}{i n+1}}
$$

(9) $\quad p_{i, k}^{(h)}=l_{i, k}^{(h)},(i-h)(h-h) \gtrless 0 ; \quad y_{i . k}^{(i)}=l_{i, k}^{(i)}-\frac{\mathrm{I}}{m+1} \frac{\partial \log \delta}{\partial x_{k}}$,

$$
p_{h h}^{(h)}=b_{h . h}^{(h)}-\frac{2}{m+1} \frac{\partial \log \delta}{\partial x_{h}},
$$

permettent de les représenter sous une forme plus commode et changent les équations (I) en d'autres semblables,

$$
\frac{d^{2} x_{h}-\sum_{(i, k)} b_{i, k}^{(h)} d x_{i} d x_{k}}{d x_{h}}=\frac{d^{2} x_{h^{\prime}}-\sum_{(i, k)} b_{i, k}^{\left(h^{\prime}\right)} d x_{i} \cdot d x_{k}}{d x_{h^{\prime}}}
$$

où figurent seuls les coefficients $b_{i . k}^{(h)}$. D'après les égalités (6) $\$$ I, ces derniers sont liés par les relations

$$
\sum_{(i)} b_{i, k}^{(i)}=0
$$


ils sont entièrement connus, si les équations (io) sont elles-mêmes données et l'on voit qu'ils ne dépendent pas du déterminant $\delta$. Il est aisé d'achever l'élimination de celui-ci entre les équations (8) et voici les relations ainsi formées,

$$
\frac{\partial f_{i . i}}{\partial x_{i^{\prime}}}=2 \sum_{(h)} b_{h, i}^{(i)} f_{i, h},\left(i-i^{\prime} \gtrless 0\right)
$$

$$
\begin{gathered}
\frac{\partial f_{i . k}}{\partial x_{i^{\prime}}}=\sum_{(h)}\left(b_{h . i^{\prime}}^{(k)} f_{i, h}+b_{h . i^{\prime}}^{(i)} f_{h . k}\right),\left[(i-k)\left(i^{\prime}-k\right)\left(i-i^{\prime}\right) \gtrless 0\right], \\
\frac{\partial f_{i . i}}{\partial x_{i}}-2 \frac{\partial f_{i, k}}{\partial x_{k}}=2 \sum_{(h)}\left[b_{i . h}^{(i)} f_{i, h}-b_{k . h}^{(k)} f_{i, h}-b_{h . k}^{(i)} f_{h . k}\right]
\end{gathered}
$$

leur nombre est $\frac{m(m-1)(m+2)}{2}$; jointes aux expressions des $m$ dérivées partielles, $\frac{\partial \log \delta}{\partial x_{k}}$, elles seraient équivalentes aux équations (8). De plus, à l'égard des quantités $f_{i, i}, f_{i . k}$, le système (12) est purement linéaire.

Ainsi et, comme conclusions des remarques précédentes, pour que les équations ( 10 ) appartiennent à un problème de dynamique, il faut et il suffit que les équations (12) admettent une solution. Lorsqu'il en est ainsi, la forme quadratique correspondante

$$
2 T d t^{2}=\sum_{(i, k)} e_{i, k} d x_{i} d x_{k}
$$

se calcule sans difficulté, car les déterminants symétriques

$$
\left\|E_{i . k}\right\|,\left\|e_{i . k}\right\|
$$

vérifient évidemment l'identité

$$
\left\|E_{i . k}\right\| \cdot\left\|e_{i . k}\right\|=1
$$

par suite, d'après l'égalité (12) § I,

$$
\left\|E_{i . k}\right\|=\frac{\mathrm{I}}{c \delta^{2}}
$$

et, faisant égale à I la constante $c$, qüil est inutile de laisser arbitraire

$$
\left\|f_{i . k}\right\|=o^{\frac{-2}{m+1}}
$$


D'ailleurs

$$
e_{i, k}=\frac{\mathrm{I}}{\left\|E_{i^{\prime}, k^{\prime}}\right\|} \cdot \frac{\partial\left\|E_{i, k^{\prime}}\right\|}{\partial E_{i . k}}
$$

c'est à dire

$$
e_{t, k}=\frac{\frac{2}{\sqrt{m+1}}}{\left\|f_{i^{\prime}, k^{\prime}}\right\|} \frac{\partial\left\|f_{i^{\prime}, k^{\prime}}\right\|}{\partial f_{i, k}}=\frac{\partial\left\|f_{i^{\prime}, k^{\prime}}\right\|}{\left\|f_{i^{\prime}, k^{\prime}}\right\|^{2} \partial f_{i, k}},
$$

de sorte que la forme (13) s'exprime de cette manière

$$
2 T d t^{2}=\frac{\mathrm{I}}{\left\|f_{i^{\prime}, k^{\prime}}\right\|^{2}} \sum_{(i, k)} \frac{\partial\left\|f_{i^{\prime}, k^{\prime}}\right\|}{\partial f_{i, k}} d x_{i} d x_{k}
$$

On a vu que, les équations ( $\mathrm{r}$ ) étant données, le système linéaire associé ne l'est pas cornplètement: sa détermination n'est achevće que si l'on connait une certaine fonction caractéristique $\delta$, dont le choix d'ailleurs est à volonté.

Ceci rappelé, j'imagine que les identités (1 2) possèdent, non plus une, mais deux solutions distinctes et j'accentue, pour les distinguer, les quantités qui se rattachent à la seconde. Il existe alors deux systèmes linéaires, associés aux équations (Io) et admettant une intégrale quadratique, quelles que soient les liaisons établies entre les $x_{i}$. L'un d'eux répond à une fonction $\delta$ définie par la formule,

$$
\grave{o}=\left\|f_{i . k}\right\|^{-\frac{m+1}{2}}
$$

l'autre à

$$
o^{\prime}=\left\|f_{i . k}^{\prime}\right\|^{-\frac{m+1}{2}} \text {. }
$$

Mais les équations (ro) sont indépendantes de $\delta$ et rien n'empêche de leur associer un système linéaire pour lequel la fonction caractéristique soit une constante. Soit $\zeta$ l'inconnue principale de ce système, en sorte que, ( $($ I)

$$
\frac{d^{2} x_{h}-\sum_{(i . h)} b_{i, k}^{(h)} d x_{i} d x_{k}}{d x_{h}}=\frac{d^{2} \zeta}{d \zeta} .
$$


Les relations (9) montrent qu'il en faut conclure

$$
\frac{d^{2} \zeta}{d \zeta}=\frac{d^{2} z}{d z}-\frac{2}{m+\mathrm{I}} d \log \delta
$$

c'est dire qu'il est permis de prendre

$$
d \zeta=\delta^{\frac{-2}{n+1}} \cdot d z=\left\|f_{i, k}\right\| d z .
$$

Or les équations (10), qui sont celles des trajectoires du mouvement étudié, s'obtiennent en joignant au système linéaire

$$
d r_{i}-\sum_{(h . k)} b_{h . k}^{(i)} r_{h} d x_{k}=0
$$

les équations suivantes

$$
\frac{d x_{i}}{d \zeta}=r_{i}
$$

et, puisque le système primitif

$$
d q_{i}-\sum_{\{(h . k)} p_{h . k}^{(i)} q_{h} d x_{k}=\mathrm{o}
$$

répondant à l'inconnue principale $z$, à la fonction caractéristique $\delta$, admet une intégrale quadratique

$$
\frac{\mathrm{I}}{\left\|f_{i^{\prime}, k^{\prime}}\right\|^{2}} \sum_{(i, k)} \frac{\partial\left\|f_{i^{\prime}, k^{\prime}}\right\|}{\partial f_{i, k}} q_{i} q_{k}=\text { constante, }
$$

le système (20) lui-même en possède une

$$
\sum_{(i, k)} \frac{\partial\left\|f_{i}^{\prime}, k^{\prime}\right\|}{\partial f_{i, k}} r_{i} r_{k}=\text { constante. }
$$

Les mêmes choses étant vraies quand l'inconnue principale est $z^{\prime}$ et la fonction caractéristique $\sigma^{2}$, il en doit admettre une seconde,

$$
\sum_{(i, k)} \frac{\partial\left\|f_{i^{\prime}, k^{\prime}}^{\prime}\right\|}{\partial f_{i . k}^{\prime}} \gamma_{i} r_{k}=\text { constante; }
$$

leur quotient,

$$
\sum_{(i, k)} \frac{\partial\left\|f_{i^{\prime}, k^{\prime}}^{\prime}\right\|}{\partial f_{i, k}^{\prime}} d x_{i} d x_{k}: \sum_{(i, k)} \frac{\partial\left\|f_{i^{\prime}, k^{\prime}}\right\|}{\partial f_{t, k}} d x_{i} d x_{k}=\text { constante, }
$$


où n'entre plus $\zeta$, est donc une intégrale des équations (Io). Il est aisé de la transformer, en $\mathrm{y}$ faisant apparaître $d z$ ou, ce qui est la mème chose, $d t$, car l'équation (23) peut se représenter ainsi

$$
\sum_{(i, k)} \frac{\partial\left\|f_{i, k^{\prime}}\right\|}{\partial f_{i, k}} d x_{i} d x_{k}=c\left\|f_{i, k^{*}}\right\|^{2} d t^{2}
$$

ce qui change (26) en une intégrale quadratique,

$$
\frac{I}{\left\|f_{i^{\prime}, k^{\prime}}\right\|^{2}} \sum_{(i, k)} \frac{\partial\left\|f_{i^{\prime}, k^{\prime}}^{\prime}\right\|}{\partial f_{i . k}^{\prime}} \frac{d x_{i}}{d t} \frac{d x_{k}}{d t}=\text { constante. }
$$

Mais il y a d'autres conclusions à déduire des relations (12). Comme elles sont linéaires, à l'égard des inconnues qu'elles renferment, s'il en existe deux solutions distinctes, les fonctions

$$
F_{i, k}=f_{i, k}^{\prime}+c f_{i, k},
$$

qui contiennent une constante arbitraire $c$, en font connaitre une solution plus générale, à laquelle s'appliquent aussi les considérations précédentes. Il y a donc en ce cas une intégrale quadratique,

$$
\frac{\mathrm{I}}{\left\|f_{i, k^{\prime}}\right\|^{2}} \sum_{(i, k)} \frac{\partial\left\|F_{i^{\prime}, k^{\prime}}\right\|}{\partial F_{i . k}} \frac{d x_{i}}{d t} \frac{d x_{k}}{d t}=\text { constante, }
$$

dont le premier membre est une fonction entière de $c$; toutes réductions faites, cette arbitraire y figure en général à la puissance $m-\mathrm{I}$.

Ainsi et pour conclure sur ce sujet: quand le système linéaire (20), associé aux équations de la dynamique, admet deux intégrales du second degré, ces équations elles-mêmes admettent en général, outre l'intégrale des forces vives, $m-\mathrm{I}$ intégrales quadratiques, coefficients des différentes puissances de $c$ dans lé premier membre de l'équation (29). Au reste, si l'on considère les mouvements qui répondent aux deux expressions

$$
\begin{aligned}
2 T d t^{2} & =\sum_{(i, k)} e_{i, k} d x_{i} d x_{k}, \\
2 T^{\prime} d t^{2} & =\sum_{(i, k)} e_{i . k}^{\prime} d x_{i} d x_{k},
\end{aligned}
$$

prises pour intégrales des forces vives, les équations (10) leur sont communes, et définissent leurs trajectoires. La question qui vient d'être étudiée 
n'est donc qu'une généralisation du problème résolu par M. Drni quand les variables sont au nombre de deux. La transformation par laquelle on peut passer de l'un de ces mouvements à l'un de ses conjugués est immédiatement en évidence. Si l'on désigne, pour le premier, par $t$, pour le second, par $t^{\prime}$, la variable qui représente le temps, l'analyse précédente montre que l'on a

$$
d t=\frac{2}{\delta^{m+1}} d \zeta, \quad d t^{\prime}=o^{\frac{2}{v^{m+1}}} d \zeta, \quad d t^{\prime}=d t\left(\frac{\delta^{\prime}}{\delta}\right)^{\frac{2}{m+1}},
$$

c'est à dire, à cause de l'égalité (15),

$$
d t^{\prime}\left\|f_{i, k}^{\prime}\right\|=d t\left\|f_{t, k}\right\|
$$

Quand

$$
\left\|f_{i . k}^{\prime}\right\|=\left\|f_{i . k}\right\|
$$

d'où suit

$$
\left\|e_{i . k}^{\prime}\right\|=\left\|e_{i . k}\right\|
$$

la relation (3 $\mathrm{I})$ se réduisant à $d t=d t^{\prime}$, les deux mouvements considérés sont confondus; en d'autres termes, il y a deux intégrales quadratiques, qui peuvent jouer le même rôle que celles des forces vives. Afin qu'un pareil cas se présente, il est évidemment nécessaire et suffisant que les équations (7) § I,

$$
\frac{\partial e_{i . k}}{\partial x_{i^{\prime}}}+\sum_{(h)}\left(p_{k . i^{\prime}}^{(h)} e_{i . h}+p_{i, i^{\prime}}^{(h)} e_{h, k}\right)=0
$$

où l'on regarde comme inconnues les $e_{i . k}$ et comme données les coefficients $p_{i . k}^{(h)}$, admettent deux solutions différentes.

L'existence de semblables cas pourrait sembler incertaine a priori, mais ils ont été obtenus d'une façon explicite, lorsque les variables $x_{i}$ sont au nombre de 3 (Note de l'auteur, Comptes rendus, avril i 89I). Quand il y a seulement deux variables, de tels mouvements ne peuvent se produire que sur les lignes géodésiques des surfaces à courbure constante. L'intégrale (26) a d'abord été signalée, pour ces cas, dans la communication qui vient d'être citée. Le théorème général a été donné, avec d'autres propositions, par M. Painlevé dans une note, insérée aux Comptes rendus de l'Académie des Sciences de Paris le I I avril 1892. Le théorème, établissant l'éxistence de plusieurs intégrales quadratiques 
et les conditions pour qu'un système d'équations (IO) appartienne à un problème de dynamique, a été énoncé sans démonstration par l'auteur de ce Mémoire, dans une note insérée aux Comptes rendus de l'Académie des Sciences le 25 avril 1892.

\section{CHA PITRE II.}

\section{4. Intégrales d'ordre supérieur à deux. Propriétés de certaines intégrales, qui forment une classe séparée.}

Un point parait très digne d'intérét dans la théorie qui vient d'être exposée: les intégrales quadratiques trouvées forment une classe, dont la considération des systèmes linéaires associés aux équations de la dynamique met en évidence les caractères tout spéciaux. Voici en effet ce qui arrive d'ordinaire, quand les équations de la dynamique admettent une intégrale du second degré,

$$
\sum_{i, k)} e_{i, k} \frac{d x_{i}}{d t} \frac{d x_{k}}{d t}=\text { constante }
$$

différente de celle des forces vives.

Le système linéaire associé est adjoint au suivant

$$
d q_{i}-\sum_{(h . k)} p_{h . k}^{(i)} q_{h} d x_{k}=0
$$

et celui-ci possède, à cause des relations $\left(5^{\prime}\right) \S \mathrm{I}$, une intégrale du second degré,

$$
\sum_{(i, k)} e_{i, k} q_{i} q_{k}=\text { constante, }
$$

mais seulement, lorsqu'on suppose entre les $x_{i}$ les liaisons définies par les équations du mouvement,

$$
\frac{d^{2} x_{h}-\sum_{(i, k)} p_{i, k}^{(h)} d x_{i} d x_{k}}{d x_{h}}=\frac{d^{2} x_{h^{\prime}}-\sum_{(i, k)} p_{i . k}^{\left(h^{\prime}\right)} d x_{i} d x_{k}}{d x_{h^{\prime}}} .
$$


Tout autre est le cas étudié; l'intégrale (2) appartient alors au système (1), quelles que soient les liaisons établies entre les $x_{i}$, si pourtant la fonction caractéristique est choisie comme il convient. Il serait naturel de faire, pour les intégrales d'ordre supérieur à deux, une distinction de cette espèce, en sorte qu'on est conduit à demander quels sont les problèmes de dynamique ou les problèmes plus généraux répondant à des équations différentielles de mème aspect, pour lesquels l'un des systèmes linéaires ( $\mathrm{I}$ ) possède une intégrale d'ordre quelconque $n$, indépendante des liaisons établies entre les $x_{i}$. La réponse à cette question est donnée par l'application d'un important théorème de M. Darboux.

Soit $R$ cette intégrale d'ordre $n$, que je suppose entière et homogène à l'égard des quantités $q_{i}$. Quand les variables $x_{i}$ sont exprimées en fonction de l'une d'entre clles, d'une manière quelconque mais définitive, le système linéaire (1) ne peut admettre une intégrale, telle que $R=$ constante, sans en admettre d'autres, qu'on peut construire. Pour le faire voir, représentons par $q_{i}^{(1)}, q_{i}^{(2)}, \ldots, q_{i}^{(m)}, m$ solutions distinctes des équations (I). Il est aisé de calculer. le déterminant $\left\|q_{i}^{(h)}\right\|$, car, en tenant compte de (r), sa différentielle est donnée par la formule

en conséquence,

$$
d\left\|q_{i}^{(h)}\right\|=\sum_{\left(i^{\prime} \cdot k\right)} p_{i^{\prime}, k}^{\left(i^{\prime}\right)}\left\|q_{i}^{(h)}\right\| d x_{k}
$$

$$
\left\|q_{i}^{(h)}\right\|=\frac{c}{\delta}
$$

$c$ désignant une constante arbitraire; d'ailleurs $\boldsymbol{o}$ est une fonction des variables $x_{i}$, traitées comme indépendantes. D'après la méthode de M. Darboux, tout covariant de $R$, où les $q_{i}$ sont prises pour les variables, multiplié par une puissance convenable de $\delta$, donne encore une intégrale des équations ( $\mathrm{I}$ ) et cette intégrale est manifestement vraie, d'après la remarque faite sur $\boldsymbol{o}$, quelles que soient les liaisons établies entre les $x_{i}$, puisqu'il en est ainsi de l'intégrale $R$.

De même, tout invariant de cette dernière, multiplié par une certaine puissance de $\delta$, doit être une constante. Comme ce produit ne peut être une intégrale, ne contenant pas les $q_{i}$, on doit, en égalant à une constante, trouver une identité satisfaite par les coefficients de l'intégrale $R$. La proposition précédente montre qu'il faut, pour l'examen de la question posée, distinguer plusieurs hypothèscs. 
$I^{\circ}$. L'intégrale $R$ est d'ordre impair: on sait qu'unc semblable forme admet en général des covariants du premier degré; le système ( $\mathrm{r}$ ) possède alors, pour un choix convenable de la fonction caractéristique, une intégrale linéaire à l'égard des $q_{i}$. Le problème correspondant est donc l'un de ceux auxquels s'appliquent en particulier les méthodes du Chapitre I. ${ }^{1}$

$2^{\circ}$. L'intégrale $R$ est d'ordre pair: Elle admet en général deux covariants quadratiques; le système (I) possède ainsi, pour un choix convenable de la fonction caractéristique, deux intégrales du second degré et, comme conséquence, le problème associé, qui est toujours un problème de dynamique proprement dit, se confond avec l'un de ceux qui ont été examinés.

$3^{\circ}$. L'intégrale $R$ est exceptionnelle: ses covariants, soit linéaires, soit quadratiques, s'évanouissent ou bien ne constituent pas des intégrales différentes de $R$. Ces cas, s"ils existent, sont les seuls qui puissent donner lieu à des recherches nouvelles, analogues à celles dont le Chapitre I fournit les éléments et cependant relatives à des problèmes essentiellement distincts.

\section{§ 5. Quelques solutions du problème de la conservation des trajectoires.}

Les problèmes de dynamique, susceptibles d'être transformés sans altération des trajectoires, s'obtiennent, d'après ce qui précède, en exprimant que les relations (12) $\$ 3$ admettent deux solutions. Voici les cas les plus simples pour lesquels cette circonstance se présente.

Considérons une forme quadratique,

$$
2 T d t^{2}=\alpha_{1}^{2} d x_{1}^{2}+\alpha_{2}^{2} d x_{2}^{2}+\ldots+\alpha_{m}^{2} d x_{n}^{2},
$$

où n'èntrent que les carrés des différentielles et proposons-nous de choisir pour $\alpha_{1}, \ldots, \alpha_{i n}$, des expressions telles,

$\Upsilon^{\circ}$ que les identités (12) $\S 3$, construites à l'aide de la forme (1), soient aussi satisfaites par une seconde forme quadratique,

$2^{\circ}$ que cette dernière ne renferme, comme la précédente, que les carrés des différentielles.

' Il est d’ailleurs possible d'obtenir tous les cas de cette espèce, donnés par une formule explicite. 
Sur les équations de la dynamique.

Or reconnait d'abord que, dans le problème de dynamique attaché à la forme (I). les coefficients $b_{i . k}^{(h)}$ sont déterminés par les formules

$$
b_{i . k}^{(i)}=-\frac{\partial \log \left(\alpha_{i} \varphi\right)}{\partial x_{k}}, \quad b_{i . i}^{(i)}=-\frac{\partial \log \left(\alpha_{i} \varphi^{2}\right)}{\partial x_{i}}, \quad b_{h . h}^{(i)}=\frac{\alpha_{h}^{2}}{\alpha_{i}^{2}} \frac{\partial \log \alpha_{h}}{\partial x_{i}},
$$

$$
b_{i, k}^{(h)}=0, \quad(i-k)(k-h)(i-h) \gtrless 0, \quad \varphi=\delta^{\frac{-1}{m+1}}=\left(\alpha_{1} \alpha_{2} \ldots \alpha_{m}\right)^{\frac{-1}{m+1}},
$$

en sorte que, pour la seconde forme quadratique, dont l'existence est supposée, les fonctions $f_{i . k}, f_{i . i}$, doivent vérifier les relations suivantes

$$
\begin{gathered}
\frac{\partial\left(f_{i . i} \alpha_{i}^{2} \varphi^{2}\right)}{\partial x_{h}}=2 \alpha_{h}^{2} \varphi^{2} \cdot f_{i . h} \frac{\partial \log \alpha_{h}}{\partial x_{i}}, \quad \frac{\partial\left(f_{i . k} \alpha_{i} \alpha_{k} \varphi^{2}\right)}{\partial x_{h}}=\frac{\alpha_{i} \alpha_{h} \varphi^{2}}{\alpha_{k}} f_{i . h} \frac{\partial \alpha_{h}}{\partial x_{k}}+\frac{\alpha_{h} \alpha_{k} \varphi^{2}}{\alpha_{i}} \frac{\partial \alpha_{h}}{\partial x_{i}} f_{h . k}, \\
\frac{\partial\left(\alpha_{i}^{2} \varphi^{2} f_{i . i}\right)}{\partial x_{i}}-2 \alpha_{i}^{2} \varphi^{2} f_{i . i} \frac{\partial \log \alpha_{h}}{\partial x_{i}}-2 \frac{\alpha_{i}}{\alpha_{h}} \frac{\partial\left(\alpha_{i} \alpha_{h} \varphi^{2} f_{i, h}\right)}{\partial x_{h}}+2 \alpha_{h}^{2} f_{h . h} \varphi^{2} \frac{\partial \log \alpha_{h}}{\partial x_{i}} \\
+\frac{2 \alpha_{i}}{\alpha_{h}} \alpha_{i} \alpha_{h} f_{i . h} \frac{\partial \log \alpha_{i}}{\partial x_{h}}-2 \sum_{\left(h^{\prime}\right)} \frac{\alpha_{i}}{\alpha_{h^{\prime}}} \varphi^{2} f_{i, h^{\prime}} \frac{\partial}{\partial x_{h}} \log \left(\frac{\alpha_{h}}{\alpha_{i}}\right)=0, \\
(i-h)(i-h) \gtrless 0, \quad\left(i-h^{\prime}\right)\left(k-h^{\prime}\right) \gtrless 0 ;
\end{gathered}
$$

de plus, $f_{i, k}=0$, dès que $i$ n'est pas égal à $k$. Le produit,

$$
f_{i . i} \alpha_{i}^{2} \varphi^{2}=\phi_{i}
$$

est donc une fonction de la seule variable $x_{i}$, en vertu de la première équation (3); la deuxième équation du même groupe est déjà satisfaite et la troisième peut se représenter ainsi

$$
\frac{\partial \psi_{i}}{\partial x_{i}}=2\left(\psi_{i}-\phi_{h}\right) \frac{\partial \log \alpha_{h}}{\partial x_{i}},
$$

ce qui exige

$$
\alpha_{h}^{2}=\prod_{\left(h^{\prime} \gtrless h\right)}\left(\psi_{h}-\phi_{h^{\prime}}\right) F_{h}\left(x_{h}\right),
$$

ou bien, après une transformation évidente,

$$
\alpha_{h}^{2}=\prod_{\left(h^{\prime} \geqslant h\right)}\left(\psi_{h}-\phi_{h^{\prime}}\right)
$$

Les fonctions $\phi_{h}$ sont arbitraires, chacune d'elles dépend de la seule vaActa mathematica. 19. Imprimê le 20 arril 1895. 
R. Liouville.

riable $x_{h}$ et le produit s'étend à toutes les combinaisons dans lesquelles $h^{\prime}$ est différent de $h$. Comme d'ailleurs

$$
f_{i . i}=\frac{\phi_{i}}{\alpha_{i}^{2} \varphi^{2}}
$$

les coefficients de la forme quadratique $\sum_{(i)} e_{i . i} d x_{i}^{2}$, conjuguée de (I), sont donnés par les formules

$$
e_{i, i}=\frac{1}{f_{i . i}} \frac{\left(\alpha_{1} \alpha_{2} \ldots \alpha_{m}\right)^{\frac{2}{m+1}}}{\psi_{1}^{\prime} \psi_{2}^{\prime} \ldots \psi_{m}}=\frac{\mathrm{I}}{\psi_{1}^{\prime} \psi_{2}^{\prime} \ldots \psi_{1 m}^{\phi_{m}}} \frac{\alpha_{i}^{2}}{\psi_{i}^{\prime}} .
$$

Le problème de dynamique attaché à la forme quadratique (I) admet $m$ - 1 intégrales du second degré, conformément au théorème général; ces intégrales sont distinctes et comprises dans l'équation suivante

$$
\sum_{(i)} \frac{\left(\psi_{1}+c\right) \ldots\left(\phi_{m}+c\right)}{\phi_{i}+c} \alpha_{i}^{2}\left(\frac{d x_{i}}{d t}\right)^{2}=\text { constante, }
$$

où la constante $c$ est arbitraire. Entre ces résultats et ceux qui se rapportent à un cas bien connu, pour lequel les équations de la dynamique admettent aussi $m$ intégrales du second degré, l'analogie n'est quapparente; les relations $\left(5^{\prime}\right)$ font connaitre en réalité un cas entièrement nouveau et répondant $\dot{a}$ des expressions tout aussi simples.

\$ 6. Transformation servant à déduire, d'une solution connue du" problème, une nutve solution, pour laquelle le nombrc des variables est plus grand.

Je considère le système linéaire associé aux équations de la dynamique et je conserve les notations déjà employées; je suppose que les $x_{i}$ subissent une variation $\partial x_{i} ; z$ et $z^{(h)}$ éprouvent une variation corres. pondante $\delta z, \partial z^{h)}$ et je pose

$$
\partial z=\zeta, \quad \partial x_{i}=x_{m+i}, \quad \partial z^{(h)}=\zeta^{(h)}, \quad z^{(h)}=\zeta^{(m+h)} ;
$$

du système linéaire

$$
d z-\sum_{(h)} z^{(h)} d x_{h}=0, \quad d z^{(i)}+\sum_{(h, k)} p_{i, k}^{(h)} z^{(h)} d x_{k}=0,
$$


il résulte immédiatement celui-ci

$$
\begin{gathered}
d \zeta-\sum_{(h)}\left(\zeta^{(h)} d x_{h}+\zeta^{(m+h)} d x_{m+h}\right)=0 \\
d \zeta^{(i)}+\sum_{(h, k)}\left[p_{i . k}^{(h)} \zeta^{(h)} d x_{k}+p_{i . k}^{(h)} \zeta^{(m+h)} d x_{m+k}+\sum_{\left(h^{\prime}\right)} \zeta^{(m+h)}\left(x_{m+h^{\prime}} \frac{\partial p_{i . k}^{(h)}}{\partial x_{h^{\prime}}}\right) d x_{k}\right]=0 .
\end{gathered}
$$

Or il est de même espèce que la premier, mais le nombre des variables y est double; les équations différentielles qui lui sont associées sont

$I^{\circ}$ celles qui l'étaient aux équations (2),

$2^{\circ}$ les suivantes

$$
\begin{gathered}
d x_{m+h} d^{2} \zeta-d \zeta d^{2} x_{m+h} \\
+\sum_{(i, k)} d \zeta\left[p_{i . k}^{(h)}\left(d x_{i} d x_{m+k}+d x_{k} d x_{m+i}\right)+\sum_{\left(h^{\prime}\right) !} x_{m+h^{\prime}} \frac{\partial y_{i . k}^{(h)}}{\partial x_{h^{\prime}}} d x_{i} d x_{k}\right]=0
\end{gathered}
$$

qui peuvent être regardées comme représentant les variations des premières. De même, en posant $\delta q_{i}=q_{m+i}$, on déduit, du système adjoint à (2),

$$
d q_{i}-\sum_{(h . k)} p_{h . k}^{(i)} q_{h} d x_{k}=\mathrm{o}
$$

celui-ci

$$
d q_{m+i}=\sum_{(h . k)}\left[p_{h . k}^{(i)}\left(q_{h} d x_{m+k}+q_{m+h} d x_{k}\right)+\sum_{\left(h^{\prime}\right)} \frac{\partial p_{h . k}^{(i)}}{\partial x_{h^{\prime}}} x_{m+h^{\prime}} q_{h} d x_{k}\right],
$$

qui est encore de même espèce. Je dis que chaque couple de formes quadratiques conjuguées, c'est à dire correspondant aux mèmes trajectoires, donne le moyen d'en construire une autre, où le nombre des variables est double. Pour le montrer, prenons un paramètre quelconque $a$, de sorte que $\partial x_{i}=\frac{\partial x_{i}}{\partial \alpha} d \alpha$. Supposons, de plus, que le problème de dynamique associé aux équations (2) corresponde à deux formes quadratiques conjuguées: le système $\left(2^{\prime}\right)$ admet une intégrale du second degré,

$$
\sum_{(i, k)} e_{f, k} q_{i} q_{k}=\text { constante, }
$$

donnée par la première des formes considérées et de laquelle on conclut

$$
\sum_{(i, k)} e_{i, k}\left(q_{i} \partial q_{k}+q_{k} \partial q_{i}\right)+\sum_{i, k, k, h)} \frac{\partial e_{i \cdot k}}{\partial x_{h}} q_{i} q_{k} \partial x_{h}=\text { constante }
$$


c'est à dire

$$
\sum_{(i, k)}\left[e_{i, k}\left(q_{i} q_{n+k}+q_{k} q_{m+i}\right)+\sum_{(h)}\left(x_{m+h} \frac{\partial e_{i, k}}{\partial x_{h}}\right) q_{i} q_{k}\right]=\text { constante. }
$$

Voilà donc, pour le système $\left(3^{\prime}\right)$, une intégrale quadratique. Mais ce qui vient d'être dit de la première forme attachée au problème de dynamique considéré, peut être dit de sa conjuguée,

$$
\sum_{(i, k)} e_{i, k}^{\prime} q_{i} q_{k}=\text { constante. }
$$

A cette dernière correspond donc une forme, semblable à (6),

$$
\sum_{\langle i, k)}\left[e_{i . k}^{\prime}\left(q_{i} q_{m+k}+q_{k} q_{m+i}\right)+q_{i} q_{k} \sum_{(k)} x_{m+h} \frac{\partial e_{i . k}^{\prime}}{\partial x_{h}}\right]=\text { constante, }
$$

et constituant avec cette dernière une couple de formes conjuguées; c'est ce qu'il s'agissait d'établir. On remarquera que la construction des formes (6) et (8) n'exige aucun calcul et qu'une moitié des variables entre au premier degré dans leurs coefficients.

\section{§ \%. Intégrales rationnelles. Propviété des équations différentielles de même aspect que celles de la dynamique.}

Bien que la remarque, très simple, contenue dans ce paragraphe, ne se rattache pas d'une façon immédiate aux questions traitées dans ce chapitre et demeure en quelque sorte isolée,' il m'a semblé utile de la présenter, avant de mettre fin à cette étude sommaire des problèmes pour lesquels il y a une intégrale des forces vives. Je suppose donc qu'il s'agisse d'étudier un mouvement pour lequel l'intégrale des forces vives existe et la valeur de l'énergie est une constante donnée. Les notations ordinaires étant admises, soient $R$ et $S$ des fonctions homogènes et entières des dérivées $\frac{d x_{i}}{d t}, \frac{R}{S}$ une intégrale rationnelle, d'où suit que $R=0, S=0$ sont des équations invariantes. Construisons le système linéaire associé aux équations du mouvement et le système adjoint

$$
d q_{i}-\sum_{(h . k)} p_{h . k}^{(i)} q_{h} d x_{k}=0
$$


On sait, $(\S \mathrm{I})$, que l'on peut remplacer $\frac{d x_{i}}{d t}$ par $q_{i}$ dans chacune des fonctions $R$ et $S$ et, puisqu'elles donnent des équations invariantes, il faut avoir

$$
\frac{d R}{d t}=\sum_{(i)} \frac{\partial R}{\partial q_{i}} \frac{d q_{i}}{d t}+\sum_{(i)} q_{i} \frac{\partial R}{\partial x_{i}}=\lambda R
$$

$\lambda$ étant une fonction linéaire des $q_{i}$. Or, d'après ( 1 ),

$$
\frac{d q_{i}}{d t}=\sum_{\left(h . h^{\prime}\right.} p_{h h^{\prime}}^{(i)} q_{h} q_{h^{\prime}}
$$

et, si

$$
\lambda=\sum_{(h)} \lambda_{h} q_{h}
$$

il en résulte

$$
\sum_{(i)}\left[q_{i} \frac{\partial R}{\partial x_{i}}+\sum_{\left(h . h^{\prime}\right)} \frac{\partial R}{\partial q_{i}} p_{h . h^{\prime}}^{(i)} q_{h} q_{h^{\prime}}\right]=R \sum_{(h)} \lambda_{h} q_{h}
$$

Je représente par $n$ le degré de $R$ à l'égard des $q_{i}$, de sorte que

$$
R=\frac{1}{n} \sum_{(i)} q_{i} \frac{\partial R}{\partial q_{i}} .
$$

Grâce à cette identité, voici, sous une autre forme, l'équation (5),

$$
\sum_{(i)}\left[q_{i} \frac{\partial R}{\partial x_{i}}+\sum_{\left(h, h^{\prime}\right)} p_{h, h^{\prime}}^{(i)} \frac{\partial R}{\partial q_{i}} q_{h} q_{h^{\prime}}-\sum_{(h)} q_{i} q_{h} \frac{\lambda_{h}}{n} \frac{\partial R}{\partial q_{i}}\right]=0
$$

Mais, après avoir posé

(7) $p_{i . h}^{(i)}-\frac{\lambda_{h}}{2 n}=b_{i . h}^{(i)}, \quad p_{h . h}^{(h)}-\frac{\lambda_{h}}{n}=b_{h . h}^{(h)}, \quad p_{h . h^{\prime}}^{(i)}=b_{h . h}^{(i)} ; \quad(h-i)\left(h^{\prime}-i\right) \gtrless 0$, l'égalité (6) devient

$$
\sum_{(i)}\left[q_{i} \frac{\partial R}{\partial x_{i}}+\sum_{\left(h . h^{\prime}\right)} b_{h . h^{\prime}}^{(i)} \frac{\partial R}{\partial q_{i}} q_{h} q_{h^{\prime}}\right]=0
$$

comme conséquence, les équations différentielles associées au système linéaire

$$
d z-\sum_{(h)} z^{(h)} d x_{h}=0, \quad d z^{(i)}+\sum_{(h . k)} b_{i . k}^{(h)} z^{(h)} d x_{k}=0
$$


R. Liouville.

admettent $R$ pour intégrale entière et homogène; mais celui-ci n'a plus d'une façon nécessaire une intégrale $\mathrm{du}$ second degré, rien ne prouve même que, sans exception,

$$
d \log \hat{\sigma}=-\sum_{(i, k)} b_{i k}^{(i)} d x_{k}
$$

soit une différentielle exacte.

La conclusion est qu'à chaque problème de dynamique possédant une intégrale rationnelle, on peut faire correspondre un ensemble d'équations différentielles, de même aspect que les équations de la dynamique et possédant une intégrale entière.

\section{CHAPITRE III.}

\$ 8. Equations générales de la mécanique, quand l'intégrale des forces vives n'existe pas, ou, celle-ci ayant lieu, quand la constante le l'energie doit rester arbitraire. Systèmes linéaires associés.

Equations différentielles de même aspect que celles de la mecanique. Transformations avec conservation des trajectoires.

Soit un système matériel, soumis à des forces quelconques $X_{i}$, que je suppose fonctions des seules quantités $x_{1}, x_{2}, \ldots, x_{m}$. Représentons encore par

$$
T=\frac{\mathrm{I}}{2} \sum_{(i . k)} e_{i . k} \frac{d x_{i}}{d t} \frac{d x_{k}}{d t}
$$

la demi-somme des forces vives: voici les équations du mouvement,

$$
\sum_{(i)} e_{i . k} \frac{d^{2} x_{k}}{d t^{2}}+\sum_{\left(h . h^{\prime}\right)}\left(\frac{\partial f_{i, h^{\prime}}}{\partial x_{h}}-\frac{\mathrm{I}}{2} \frac{\partial e_{h . h^{\prime}}}{\partial x_{i}}\right) \frac{d x_{h}}{d t} \frac{d x_{h^{\prime}}}{d t}-X_{i}=0
$$

auxquelles il est clair qu'on peut donner la forme

$$
d x_{h} d^{2} t-d t d^{2} x_{h}+d t \cdot \sum_{(i, k)} p_{l . k}^{(h)} d x_{i} d x_{k}+p_{h} d t^{3}=0
$$


en déterminant les $p_{i, k}^{(h)}$ par les relations déjà employées

$$
2 \sum_{(k)} p_{h . h^{\prime}}^{(k)} e_{i . k}+\frac{\partial e_{i, h^{\prime}}}{\partial x_{h h}}+\frac{\partial e_{i . h}}{\partial x_{h^{\prime}}}-\frac{\partial e_{h . h^{\prime}}}{\partial x_{i}}=0
$$

et, de plus, les $p_{k}$ par les suivantes

$$
\sum_{(k)} e_{i . k} p_{k}=X_{i}
$$

Considérons un système d'équations différentielles linéaires,

$$
\begin{aligned}
d z-\sum_{(h)} z^{(h)} d x_{h} & =0, \quad(i, k, h \leqq m+\mathrm{I}) \\
d z^{(i)}+\sum_{(h, k)} p_{i . k}^{(h)} z^{(h)} d x_{k} & =0,
\end{aligned}
$$

dans lequel les variables sont $x_{1}, x_{2}, \ldots, x_{m}$ et $x_{m+1}=t$, dans lequel en outre on suppose

$$
\begin{gathered}
p_{i . m+1}^{\left(h^{\prime}\right)}=p_{i . i^{\prime}}^{(m+1)}=0, \quad\left(\mathrm{O}<i \leqq m, 0<i^{\prime} \leqq m, 0<h^{\prime} \leqq m+\mathrm{I}\right) \\
p_{n+1 . m+1}^{(h)}=p_{h}, \quad(\mathrm{o}<h \leqq m), \quad p_{m+1, n+1}^{(m+1)}=0 .
\end{gathered}
$$

Les relations (2) dépendent du système précédent comme, au $§$ I, les équations (4) dépendaient du système (2). Nous dirons encore que les équations linéaires (5) sont associées aux relations (2). Cette définition n'exige nullement, on le voit, qu'il y ait une forme quadratique $T$ dont les coefficients soient liés aux $p_{i, k}^{(h)}$ par les identités (3). Si cette forme n'existe pas, les équations associées à (5) conservent le même aspect que celles de la mécanique, elles sont cependant beaucoup plus générales, car

$I^{\circ}$ les $p_{k}$ demeurent des fonctions quelconques de $x_{1}, x_{2}, \ldots, x_{m}$

$2^{\circ}$ les $p_{i . k}^{(h)}$ ne sont plus assujettis qu'à ces conditions

$$
\sum_{(i)} p_{i . k}^{(i)}=-\frac{\partial \log \delta}{\partial x_{k}}
$$

toujours supposées jusqu’à présent.

Elimination faite de la variable $x_{m+1}$, il $\mathrm{y}$ a, entre les $x_{i}, m-2$ équations du second ordre, qu'on peut représenter ainsi 


$$
\begin{aligned}
\left(p_{h^{\prime}} d x_{h^{\prime \prime}}-p_{h^{\prime}} d x_{h^{\prime}}\right)\left(d^{2} x_{h^{\prime}}-\sum_{(i . k)} p_{i . k}^{(h)} d x_{i} d x_{k}\right) \\
+\left(p_{h^{\prime \prime}} d x_{h}-p_{h} d x_{h^{\prime \prime}}\right)\left(d^{2} x_{h^{\prime}}-\sum_{(i . k)} p_{i . k}^{\left(h^{\prime}\right)} d x_{i} d x_{k}\right) \\
+\left(p_{h} d x_{h^{\prime}}-p_{h^{\prime}} d x_{h}\right)\left(d^{2} x_{h^{\prime \prime}}-\sum_{(i . k)} p_{i, k}^{\left(h^{\prime \prime}\right)} d x_{i} d x_{k}\right)=0
\end{aligned}
$$

ou, d'une façon plus simple, $(\$ 2)$,

$$
\left|\begin{array}{lll}
p_{h} & d x_{h} & D d x_{h} \\
p_{h^{\prime}} & d x_{h^{\prime}} & D d x_{h^{\prime}} \\
p_{h^{\prime \prime}} & d x_{h^{\prime \prime}} & D d x_{h^{\prime \prime}}
\end{array}\right|=0 ;
$$

il y en a une dernière, d'ordre 3, c'est la suivante

$$
\begin{gathered}
d \log \left[d x_{h} D d x_{h^{\prime}}-d x_{h^{\prime}} D d x_{h}\right] \\
=d \log \left(p_{h^{\prime}} d x_{h}-p_{h} d x_{h^{\prime}}\right)+\frac{2\left[p_{h} D d x_{h^{\prime}}-p_{h^{\prime}} D d x_{h}\right]}{p_{h} d x_{h^{\prime}}-p_{h^{\prime}} d x_{h}} .
\end{gathered}
$$

Les relations (8') et (9) sont ce que j'appellerai les équations des trajectoires.

Imaginons qu’il existe un second système linéaire, $\left(5^{\prime}\right)$, pareil ’̀ (5), qui corresponde aux mêmes trajectoires et distinguons par un accent toutes les quantités qui s'y rattachent. Soient d'ailleurs

$$
d \zeta=o^{\frac{2}{m+1}} d z, \quad d \zeta^{\prime}=o^{\frac{2}{m+1}} d z^{\prime} .
$$

$\mathrm{Si}$, dans les équations associées aux systèmes (5) et $\left(5^{\prime}\right)$ on remplace $z$ et $z^{\prime}$, respectivement, par $\zeta, \zeta^{\prime}$, elles conservent la même forme, mais leurs coefficients deviennent

$$
\begin{aligned}
& b_{i . k}^{(i)}=p_{i . k}^{(i)}+\frac{\mathrm{I}}{m+\mathrm{I}} \frac{\partial \log \delta}{\partial x_{k}}, \quad b_{i . k}^{(h)}=p_{i . k}^{(h)}, \quad b_{i . i}^{(i)}=p_{i . i}^{(i)}+\frac{2}{m+\mathrm{I}} \frac{\partial \log \delta}{\partial x_{i}}, \\
& \text { (I I) } \quad(i-k \gtrless 0), \quad(i-h)(k-h) \gtrless 0, \quad(i, h, k \leqq m) \\
& b_{i . k}^{\prime(i)}=p_{i . k}^{\prime(i)}+\frac{\mathrm{I}}{m+\mathrm{I}} \frac{\partial \log \delta^{\prime}}{\partial x_{k}}, \quad b_{i . k}^{\prime(h)}=p_{i . k}^{\prime(h)}, \quad b_{i . i}^{\prime(i)}=p_{i . i}^{\prime(i)}+\frac{2}{m+\mathrm{I}} \frac{\partial \log \delta^{\prime}}{\partial x_{i}} ; \\
& b_{h}=p_{h} \cdot \frac{2}{o^{m+1}}, \quad b_{h}^{\prime}=p_{h}^{\prime} \cdot o^{\frac{2}{\prime m+1}},
\end{aligned}
$$


en sorte que ces deux relations,

$$
\text { (1 3) } \frac{d^{2} x_{h}-\sum_{(i . k)} b_{i . k}^{(h)} d x_{i} d x_{k}-b_{h} d \zeta^{2}}{d x_{h}}=\frac{d^{\prime} \zeta}{d \zeta}, \frac{d^{2} x_{h}-\sum_{(i k)} b_{i . k}^{(h)} d x_{i} d x_{k}-b_{h}^{\prime} d \zeta^{\prime 2}}{d x_{h}}=\frac{d^{2} \xi^{\prime}}{d \zeta^{\prime}}
$$

sont vérifiées à la fois, quel que soit l'indice $h$. J'en déduis d'abord

$$
\left\{\sum_{(i k)}\left(b_{i . k}^{\prime(h)}-b_{i . k}^{(h)}\right) d x_{i} d x_{k}+b_{h}^{\prime} d \zeta^{\prime 2}-b_{h} d \zeta^{2}\right\}=d x_{h}\left(\frac{d^{2} \zeta}{d \zeta}-\frac{d^{2} \zeta^{\prime}}{d \zeta^{\prime}}\right)
$$

mais l'une des équations associées au système (5) était, d'après (6),

$$
d x_{m+1} d^{2} z-d z d^{2} x_{m+1}=0
$$

et l'on en conclut

$$
d x_{m+1}=c 0^{\frac{-2}{m+1}} d \zeta
$$

la constante $c$ étant arbitraire; il y a une égalité analogue pour $d \zeta^{\prime}$. Dans l'un des deux problèmes conjugués, par exemple le premier, je puis regarder $c$ comme choisie d'une façon definitive, car c'est achever la détermination de l'inconnue auxiliaire $d \zeta$. Parmi les relations (14), j'en considère trois, qui répondent à des indices différents et j'élimine entre elles

$$
d \zeta^{\prime 2}, \frac{d^{2} \zeta^{\prime}}{d \zeta^{\prime}}-\frac{d^{2} \zeta}{d \zeta}
$$

Le premier membre de l'équation obtenue est la somme de trois quantités semblables à celle-ci

$$
\left(b_{h^{\prime}}^{\prime} d x_{h^{\prime \prime}}-b_{h^{\prime \prime}}^{\prime} d x_{h^{\prime}}\right)\left[\sum_{(i, k)}\left(b_{i . k}^{(h)}-b_{i . k}^{(h)}\right) d x_{i} d x_{k}-b_{h} d \zeta^{2}\right]
$$

et s'en déduisant par permutation circulaire des indices $h, h^{\prime}, h^{\prime \prime}$. On voit, en tenant compte de (I5), que cette équation est une identité ou une intégrale du premier problème. Elle ne contient pas d'arbitraire, c'est donc une identité.

Par suite, les rapports

$$
\frac{b_{h}^{\prime}}{b_{h}}, \frac{b_{i . k}^{\prime(h)}-b_{i . k}^{(h)}}{b_{h}}
$$

Acta mathematica. 19. Imprime le 23 avril 1895. 
que contient l'équation (1 4), ne dépendent pas de $h$, en sorte qu'on peut écrire

$$
\frac{b_{i . k}^{(h)}-b_{i . k}^{(h)}}{b_{h}}=\frac{a_{i . k}}{\beta}, \quad \frac{b_{h}^{\prime}}{b_{h}}==\frac{\beta^{\prime}}{\beta} .
$$

Mais, pour rendre cette conséquence bien évidente, il est nécessaire d'entrer dans quelques détails. Dans l'identité

$$
\left(b_{h^{\prime}}^{\prime} d x_{h^{\prime \prime}}-b_{h^{\prime \prime}}^{\prime} d x_{h^{\prime}}\right)\left[\sum_{(i . k)}\left(b_{i . k}^{\prime(h)}-b_{i . k}^{(h)}\right) d x_{i} d x_{k}-b_{h} d \zeta^{2}\right]+\ldots=0
$$

je puis annuler $d x_{h^{\prime}}, d x_{h^{\prime}}$. La proposition devient ainsi manifeste, quand l'inégalité suivante

$$
(i-h)(k-h) \gtrless 0
$$

est vérifiée. Si j’annule ensuite $d x_{h^{\prime}}$, mais non $d x_{h^{\prime \prime}}, d x_{h}$, j'obtiens les conditions

$$
b_{i . k}^{(i)}-b_{i . k}^{(i)}=b_{i . k}^{(i)}-b_{i . h}^{\left(i^{i}\right)}=\ldots \text { etc. }
$$

d'ailleurs on sait que, d'après (6),

$$
\sum_{(i)} b_{i . k}^{(i)}=\sum_{(i)} b_{i . k}^{(i)}=0
$$

Il en résulte que les différences (19), dont la somme est nulle, doivent toutes s'évanouir. Les termes qui leur correspondraient ne figurent donc pas dans l'équation (I 4) et la proposition énoncée est entièrement établie.

A cause des égalités (1 7), maintenant démontrées, les équations (14) se laissent représenter ainsi

$$
\left[\sum_{(i, k)} a_{i, k} d x_{i} d x_{k}+\beta^{\prime} d \zeta^{\prime 2}-\beta d \zeta^{2}\right] \frac{b_{h}}{\beta d x_{h}}=\frac{d^{2} \zeta}{d \zeta}-\frac{d^{2} \zeta^{\prime}}{d \zeta^{\prime}}
$$

et, devant avoir lieu quel que soit $h$, elles exigent

$$
\frac{d^{2} \zeta}{d \zeta}-\frac{d^{2} \zeta^{\prime}}{l_{\zeta}^{\zeta^{\prime}}}=0
$$

$$
\sum_{(i . k)} a_{i . k} d x_{i} d x_{k}+\beta d \zeta^{\prime 2}-\beta d \zeta^{2}=0
$$


De l'équation (22) on conclut

$$
d \zeta^{\prime}=a d \xi
$$

la constante $a$ est arbitraire et la relation (23) fait connaitre une intégrale quadratique du problème considéré. C'est ce qui justifie le théorème suivant:

Quand on peut passer du système (2) à un autre semblable, en conservant les trajectoires, il y a pour ce système une intégrale quadratique.

J'ajoute que les quantités

$$
\left[\frac{\mathrm{I}}{b_{h}} b_{i . k}^{(h)}-\frac{\mathbf{I}}{b_{h^{\prime}}} b_{i k}^{\left(h^{\prime}\right)}\right] \frac{\mathrm{I}}{\beta}
$$

sont des invariants de la transformation.

Le théorème précédent a été énoncé par M. P. Painlevé, pour le cas particulier où les équations (2) sont celles d'un problème de mécanique. On voit qu'il s'applique également aux équations, beaucoup plus générales, de même aspect.

Il y a donc entre ce théorème et celui qui a fait l'objet du $\$ 3$ une différence essentielle et qu'il importe de signaler. Alors que ce dernier, d'après les considérations même qui l'ont fait obtenir, caractérisait des équations appartenant ̀̀ un problème de dynamique, il n'en est nullement ainsi dans le cas actuel. L'existence d'une forme quadratique $T$, dont les coefficients soient liés aux $p_{i . k}^{(h)}$ par les relations (3), impose à ces dernières fonctions des conditions supplémentaires, qui ne sont point exigées pour l'existence d'une couple de systèmes (2) correspondant aux mêmes trajectoires, ni pour celle de l'intégrale quadratique (23). 